\title{
The hereditary angioedema syndromes
}

\author{
Alvin H. Schmaier
}

Department of Medicine, University Hospitals Cleveland Medical Center and Case Western Reserve University, Cleveland, Ohio, USA.

\begin{abstract}
Hereditary angioedema (HAE) is a rare genetic disorder primarily caused by mutations in the SERPINC1 gene encoding the C1 inhibitor (C1INH) that leads to plasma deficiency, resulting in recurrent attacks of severe swelling. In the current issue of the $\mathrm{JCl}$, Haslund et al. show that in a subset of patients with type I HAE, mutated C1INH encoded by HAE-causing SERPING1 acts upon wildtype (WT) C1INH in a dominant-negative manner and forms intracellular C1INH aggregates. These aggregates lead to a reduction in the levels of secreted functional C1INH, thereby manifesting in the condition that allows the disease state. Interestingly, administration of WT SERPINC1 gene is able to restore the levels of secreted C1INH, thereby opening up a novel mechanism justifying gene therapy for HAE.
\end{abstract}

\section{Hereditary angioedema and the $\mathrm{C} 1$ inhibitor}

C1 esterase inhibitor (C1 inhibitor, C1INH) is a $105 \mathrm{kDa}$ serine protease inhibitor of the SERPIN family and gene product of SERPING1. C1INH has a plasma concentration of approximately 1.5-2.0 $\mu \mathrm{M}$, a concentration sufficient to inhibit all of its target serine proteases, factor XIIa ( $\alpha$ FXIIa), plasma kallikrein (PKa), C1 esterase, plasmin, and factor XIa (FXIa). C1INH accounts for $93 \%$ inhibition of plasma $\alpha$ FXIIa and its breakdown products, and $48 \%$ inhibition of PKa. Landerman et al. originally suggested that the disorder of hereditary angioneurotic edema (HANE) results from a deficiency of an inhibitor for serum globulin permeability factor and/or PKa (1). Donaldson and Evans are credited with deducing that C1INH is the biochemical abnormality associated with HANE (2). Their discovery forever changed the name HANE to hereditary angioedema (HAE).

HAE was first recognized by Heinrich Quincke, but William Osler is credited with its first complete description $(3,4)$. It is a lifelong disorder that usually manifests at the onset of puberty and is associated with episodic instances of localized tissue swelling that can be disfiguring. In the bowel, it can be mistaken for an acute abdomen. In the larynx, it can be life-threatening due to hypoxia and suffocation. In the United States, there are about 20,000 patients with HAE. The hallmark of the disorder is localized cleavage of high-molecular-weight kininogen (HK) with bradykinin (BK) formation leading to angioedema regardless of the inciting etiology or proteins involved. Two major questions are being pursued in the field: (a) what protein deficiency and/or defect produce the angioedemas, and (b) what is (are) the inciting mechanism(s) for acute attacks of the disorder?

The vast majority of HAE patients have deficiency of (type I) and/or defects in (type II) C1INH, but there are other inciting etiologies as well (Table 1). In type I HAE, there is a deficiency in the plasma level of C1INH, but the protein present functions normally. SERPING1 on chromosome 11q12.1 has autosomal inheritance. Patients with the disorder have plasma levels of $40 \%-60 \%$ normal between attacks, with levels being lower during an attack. Although mice devoid of C1INH (serping1 $1^{-/}$) are viable (5), homozygous $\mathrm{C} 1 \mathrm{INH}$ deficiency is not seen in humans.

\section{Mutated SERPING1 gene inhibits the expression of the WT gene}

This month in the JCI, Haslund et al. from the Mikkelsen group provide important new information for the potential mechanism in patients with type I HAE (6). Haslund et al. show that in certain subtypes of type I HAE, a mutated SERPING1 gene has an inhibitory role on the expression of the WT gene (6). In their studies, the mutated SERPING1 gene had a dominant-negative action on normal C1INH expression, triggering the formation of intracellular aggregates in the endoplasmic reticulum (ER), and resulting in a reduction in the secretion of functional C1INH. The abnormal allele of C1INH produced a protein that led to abnormal protein aggregation and accumulation within the producing cell to interfere with ER expression of the protein from the normal allele. The mousetrap function of the reactive center loop (RCL) of C1INH has a driving force for it to close on a target, because when open, the serpin molecule is inherently unstable. This inherent instability of serpins also makes them prone to aggregation since, presumptively, it is another way to achieve molecular stability. In variant $\alpha 1$-antitrypsin deficiency, the accumulation of the serpin in the hepatocyte ER causes cell death and liver cirrhosis (7). However, the mechanism proposed by Haslund et al. for type I HAE is not applicable to all mutations in C1INH. Only alleles encoding full-length or near full-length C1INH proteins were shown to most likely have the dominant-negative effect. Further, the dominant-negative HAE-causing SERPING1 variants were in close proximity to the shutter domain, a region where the RCL clamps down with its target protease to produce a stable conformation and irreversible inhibition of the protease.

Type II HAE occurs less frequently than type I and is caused when the C1INH produced has an abnormal protease inhibitory function, but is produced and secreted normally from the ER. Both type I and II HAE are considered congen- 


\section{Table 1. Etiologies of hereditary angioedema syndromes}

$\begin{array}{ll}\text { Type I HAE } & \text { Deficiency of C1INH } \\ \text { Type II HAE } & \text { Defect in C1INH } \\ \text { Type III HAE } & \begin{array}{l}\text { Normal C1INH; abnormal } \alpha \text { FXIla with } \\ \text { gain-in-function mutations }\end{array} \\ \text { Other HAEs } & \text { Abnormal plasminogens } \\ & \text { Abnormal angiopoietin 1 }\end{array}$

ital. Another variety of C1INH deficiency that is acquired and uncommonly occurs is due to an antiidiotype antibody (8). In this instance, the antibody binds to the Fc portion of immunoglobulin, activating the classic complement pathway and leading to secondary $\mathrm{C} 1 \mathrm{INH}$ deficiency due to its consumption. These rare antiidiotype antibodies are usually associated with $\mathrm{B}$ cell malignancies or Hodgkin's disease.

In the newly recognized type III HAE, the symptoms and signs of the disorder are present but the patients have normal C1INH levels (9). Type III HAE patients have mutations in the proline-rich region of factor XII (e.g., T309R, T309K) (10). The T309 site is an $\mathrm{N}$-glycosylation region whose absence is associated with accelerated FXIIa coagulant activity (11). Prior investigations on

Figure 1. Angioedema-inciting mechanisms in low C1INH or protease gain-in-function states. In all cases characterized to date, angioedema is induced by excess BK delivery to vascular endothelium. With the exception of abnormal angiopoietin 1 , there are three hypotheses regarding the initiating events for an acute attack in the hereditary angioedema syndromes leading to excess BK formation. (A) Contact activation. Ambient levels of negatively charged biologic surfaces like polyphosphates (polyP), exosomes, DNA, RNA, or aggregated-denatured protein (not shown) arise, and in the presence of low C1INH allow for FXII activation to FXIla. FXIla activates prekallikrein (PK) to form plasma kallikrein (PKa) that cleaves high-molecular-weight kininogen (HK) to liberate BK and leave cleaved HK (CHK). (B) Single-chain FXII activation. When the three arginines in the light chain of FXII are mutated to alanine, there is still some proteolytic activity of this form of FXII (1/4,000th) that can activate PK to PKa in the presence or absence of polyP. (C) Prolylcarboxypeptidase. Prolylcarboxypeptidase is a membrane endothelial cell serine protease that activates PK to PKa at a low $K_{m}(9 \mathrm{nM})$. In low C1INH states and/or when PRCP is upregulated, it has the ability to activate PK to PKa to cleave $\mathrm{HK}$ and liberate BK.
FXII show that recombinant truncation mutations in its proline-rich region have accelerated coagulant activity (12).

\section{Hereditary angioedema syndromes}

In addition to the well-characterized forms of types I, II, and III HAE, additional kindreds have HAE-like illnesses with normal C1INH and FXII. Several families have mutations in plasminogen (13). One family has been described with a defect in angiopoietin 1 (14). Thus, it appears that angioedema disorders may arise from defects and/or deficiencies in a growing list of proteins. Besides C1INH loss, other proteins contribute to this disorder, and perhaps it is time to consider this group of disorders as the "hereditary angioedema syndromes" rather than a single protein disorder. However, all these disorders to date appear to be characterized by excess BK delivery to tissues.

A Contact activation

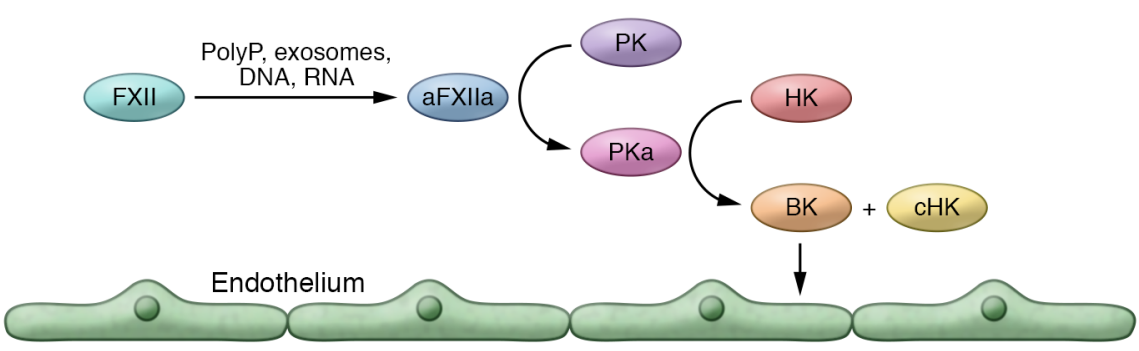

B Single-chain FXII activation

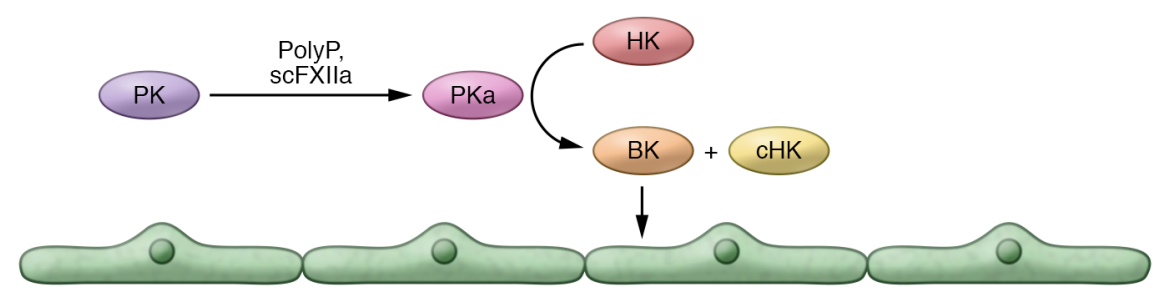

C Prolylcarboxypeptidase

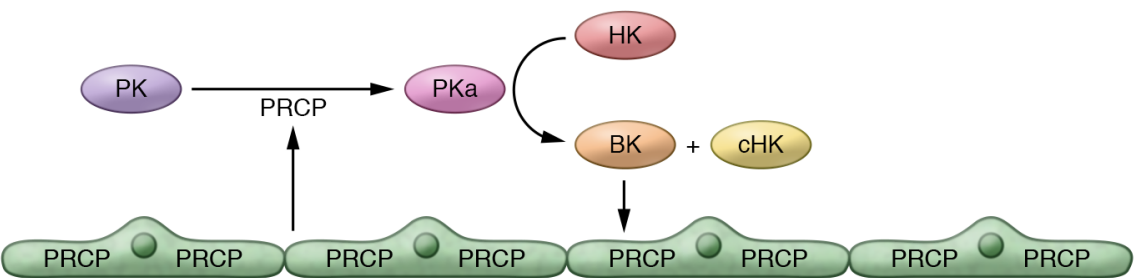

The second major unanswered question in the angioedema field is, What is the initiating etiology? Regardless of the mechanism, the majority of HAE patients are not plagued with constant attacks of angioedema. Acute attacks inciting etiologies are usually not known, but point toward associated infection, anxiety, medical interventions, and pregnancy. The actual attack mechanism(s) is also not known, but in the presence of low $\mathrm{C} 1 \mathrm{INH}$ or an abnormal protein leading to angioedema, there are at least three hypotheses (Figure 1). First, the inciting event is related to plasma contact activation. Second, zymogen forms of factor XII have intrinsic proteolytic activity that initiate prekallikrein (PK) activation. Third, the endothelial cell protease prolylcarboxypeptidase (PRCP), or others, has the ability to activate plasma PK to form PKa, which liberates BK from $\mathrm{HK}$ and indirectly activates FXII. 
Since the last decade it has been appreciated that there are circulating structures - polyphosphates (polyP), exosomes, DNA, RNA, denatured protein, and exposed collagen that have the potential to serve as platforms for contact activation (FXII autoactivation on a surface leading to PK activation with HK cleavage and BK liberation) in the intravascular compartment. In disorders such as prostate cancer and Alzheimer's disease, there is evidence for a constitutively higher state of contact activation as a result of increased circulating exosomes and denatured protein, respectively $(15,16)$. However, in attacks of HAE in type I C1INH, FXII levels are often not consumed (17). Alternatively, recent investigations using unique recombinant forms of FXII, where all active site arginines have been mutated to alanine, show that these mutant proteins still retain weaker intrinsic ability $(\sim 1 / 4,000$ th of $\alpha$ FXIIa) to activate PK both in the presence or absence of polyphosphates (18). In vivo it is as yet unknown whether zymogen FXII could be a PK activator, especially in low C1INH states. Finally, we have identified a membrane-expressed serine protease, PRCP, that has a low $K_{m}(\sim 9$ $\mathrm{nM}$ ) to activate PK when bound to endothelial cell membranes (19). Its role in intravascular PK activation in normal and low C1INH states needs further investigation.

\section{Potential for gene therapy for hereditary angioedema}

Currently, HAE has been recognized as an orphan medical disorder with much commercial development of novel therapies. In addition to various forms of $\mathrm{C} 1 \mathrm{INH}$ replacement and bradykinin $\mathrm{B} 2$ receptor blockade, intravenous monoclonal antibodies and oral nonpeptide mimetics inhibiting plasma kallikrein are entering the therapeutic arena for HAE. Likewise, plasma FXIIa inhibitors may have a role when FXIIa is squarely placed in the pathogenesis of the disorder. Medical therapy for HAE syndromes could thus be tailored for the inciting pathogenic features of the disorder. It is of interest to know that in the report by Haslund et al., normal SERPING1 transfection into cells ameliorated the block in normal C1INH secretion, suggesting that gene therapy may be useful for this type of hereditary angioedema.

In conclusion, there has been remarkable advancement in the understanding of C1INH and development in new therapeutics for HAE. Although HAE is an orphan disorder with a relatively small number of patients, these agents may prove to be valuable for the management of more common disorders like mechanical device thrombosis, venous thrombosis, and, perhaps, disorders of innate immunity where FXII contributes to their pathogenesis.

\section{Acknowledgments}

This work is supported in part from grants HL126645, AI130131, HL140902, HL144113, and HL143402 from the National Institutes of Health and BC150596P1 from the Department of Defense.

Address correspondence to: Alvin $\mathrm{H}$. Schmaier, Case Western Reserve University, 2103 Cornell Road, WRB 2-130, Cleveland, Ohio 44106-7284, USA. Phone: 216.368.0796; Email: schmaier@case.edu.

1. Landerman NS, Webster ME, Becker EL, Ratcliffe HE. Hereditary angioneurotic edema. II. Deficiency of inhibitor for serum globulin permeability factor and/or plasma kallikrein. JAllergy. 1962;33:330-341.

2. Donaldson VH, Evans RR. A biochemical abnormality in hereditary angioneurotic edema: absence of serum inhibitor of C' 1-esterase. Am J Med. 1963;35:37-44.

3. Quincke HI. Uber akutes umschriebenes Hautodem, Monatsh. Prakt Derm. 1882;1:129-131.

4. Osler W. Hereditary angioneurotic edema. Am J Med Sci.1888;95:362-366.

5. Han ED, MacFarlane RC, Mulligan AN, Scafidi J, Davis AE. Increased vascular permeability in $\mathrm{C} 1$ inhibitor-deficient mice mediated by the bradykinin type 2 receptor. J Clin Invest. 2002;109(8):1057-1063.

6. Haslund D, et al. Dominant-negative SERPING1 variants cause intracellular retention of $\mathrm{C} 1$ inhibitor in hereditary angioedema. JClin Invest. 2019;129(1):388-405.

7. Lomas DA, Mahadeva R. $\alpha 1$-Antitrypsin polymerization and the serpinopathies: pathobiology and prospects for therapy. J Clin Invest. 2002;110(11):1585-1590.

8. Geha RS, Quinti I, Austen KF, Cicardi M, Sheffer A, Rosen FS. Acquired C1-inhibitor deficiency associated with antiidiotypic antibody to monoclonal immunoglobulins. N Engl J Med. 1985;312(9):534-540.

9. Bork K, Barnstedt SE, Koch P, Traupe H. Hereditary angioedema with normal C1-inhibitor activity in women. Lancet. 2000;356(9225):213-217.

10. Dewald G, Bork K. Missense mutations in the coagulation factor XII (Hageman factor) gene in hereditary angioedema with normal C1 inhibitor. Biochem Biophys Res Commun. 2006;343(4):1286-1289.

11. Björkqvist J, et al. Defective glycosylation of coagulation factor XII underlies hereditary angioedema type III. J Clin Invest. 2015;125(8):3132-3146.

12. Citarella F, et al. Control of human coagulation by recombinant serine proteases. Blood clotting is activated by recombinant factor XII deleted of five regulatory domains. Eur J Biochem. 1992;208(1):23-30.

13. Bork K, et al. Hereditary angioedema with a mutation in the plasminogen gene. Allergy. 2018;73(2):442-450.

14. Bafunno V, et al. Mutation of the angiopoietin-1 gene (ANGPT1) associates with a new type of hereditary angioedema. J Allergy Clin Immunol. 2018;141(3):1009-1017.

15. Nickel KF, et al. The polyphosphate-factor XII pathway drives coagulation in prostate cancer-associated thrombosis. Blood. 2015;126(11):1379-1389.

16. Zamolodchikov D, Chen ZL, Conti BA, Renné T, Strickland S. Activation of the factor XII-driven contact system in Alzheimer's disease patient and mouse model plasma. Proc Natl Acad Sci U S A. 2015;112(13):4068-4073.

17. Schapira M, et al. Prekallikrein activation and high-molecular-weight kininogen consumption in hereditary angioedema. N Engl J Med. 1983;308(18):1050-1053.

18. Ivanov I, et al. Proteolytic properties of singlechain factor XII: a mechanism for triggering contact activation. Blood. 2017;129(11):1527-1537.

19. Shariat-Madar Z, Mahdi F, Schmaier AH. Identification and characterization of prolylcarboxypeptidase as an endothelial cell prekallikrein activator. J Biol Chem. 2002;277(20):17962-17969. 